$\mathrm{DF} / \mathrm{IST}-2.99$

NYU-TH/99/05/03

\title{
Nonminimal coupling and quintessence
}

\author{
Orfeu Bertolami \\ New York University \\ Department of Physics \\ Andre and Bella Meyer Hall of Physics \\ 4 Washington Place \\ New York, NY 10003-6621, USA \\ and \\ P.J. Martins \\ Departamento de Física \\ Instituto Superior Técnico, Av. Rovisco Pais \\ 1096 Lisboa Codex, Portugal
}

\begin{abstract}
Recent studies of type Ia Supernovae with redshifts up to about $z \lesssim 1$ reveal evidence for a cosmic acceleration in the expansion of the Universe. The most straightforward explanation to account for this acceleration is a cosmological constant dominating the recent history of our Universe; however, a more interesting suggestion is to consider an evolving vacuum energy. Several proposals have been put forward along these lines, most of them in the context of General Relativity. In this work we analyse the conditions under which the dynamics of a self-interacting Brans-Dicke field can account for this accelerated expansion of the Universe. We show that accelerated expanding solutions can be achieved with a quadratic self-coupling of the Brans-Dicke field and a negative coupling constant $\omega$.
\end{abstract}

\footnotetext{
${ }^{1}$ Permanent address Departamento de Física, Instituto Superior Técnico, Av. Rovisco Pais, 1096 Lisboa Codex, Portugal. E-mail: orfeu@alfa.ist.utl.pt
} 


\section{Introduction}

Type Ia Supernovae (SNe Ia) allow us, when used as standard candles, to reliably determine the cosmological parameters. Indeed, low redshift supernovae can be used to obtain the Hubble constant, $H_{0}$, while supernovae at greater distances allow probing the deceleration parameter, $q_{0}$. Recent analyses of the magnitude-redshift relation of about 50 SNe Ia with redshifts greater than $z \geq 0.35$ strongly suggest that we are living in an accelerating, low-matter density Universe $[1$ - 4]. The consistency relationship between these cosmological parameters and the luminosity distance, $d_{L}$, of a SNe is given, for $z \lesssim 1$, by $d_{L} \approx H_{0}^{-1}\left[z+\left(1-q_{0}\right) z^{2} / 2\right]$. The results obtained by different groups indicate that $[1-4]$

$$
-1 \lesssim q_{0}<0 .
$$

These values seem to favour, in the case of a flat Universe, a rather low contribution to the energy density from non-relativistic matter, say $\Omega_{M} \sim 0.3$, but on the other hand a dominant positive cosmological constant, $\Omega_{\Lambda} \sim 0.7$, as can be inferred from data [5] and consistently from the well known Friedmann model equation

$$
q_{0}=\frac{1}{2}(3 \gamma+1) \Omega_{M}-\Omega_{\Lambda}
$$

where $\Omega_{M(V)}$ denotes the energy density of matter (vacuum) in terms of the critical density, $\gamma$ stands for the constant in the matter equation of state, $p=\gamma \rho$, and observationally $-1 \leq \gamma \leq 0$ ( $\gamma=0$ for non-relativistic matter, $\gamma=1 / 3$ for relativistic matter and $\gamma=-1$ for a scalar field fluid dominated by its potential). Even though a dominant cosmological constant corresponding to $\Omega_{\Lambda} \sim 0.7$ is consistent with all observational facts (see [6] for a list of constraints and [7] for a thorough discussion) it implies a quite unnatural fine tuning of parameters if its origin lies in any known particle physics scale (see Refs. [8] for reviews and Refs. [6, 9] for possible connections with fundamental symmetries such as Lorentz invariance and string theory S-duality).

From Eq. (22) we see that a negative $q_{0}$ can also arise from a negative pressure component or 
"dark energy". This alternative calls for a scalar field endowed with a potential which can give rise to a dynamical vacuum energy, the so-called "quintessence". Suggestions along these lines were proposed long ago, although yielding, in that case, a vanishing deceleration parameter [10]. A number of quintessence models have been put forward, most of them invoking a scalar field with a very shallow potential, which until recently was overdamped by the expansion of the Universe, so that its energy density was smaller than the radiation energy density at early times [11, 12, 13]. For instance, scalar fields with an exponential type potential can, under conditions, render a negative $q_{0}$ [14, 15]. More involved possibilities include the string theory dilaton together with gaugino condensation [16], an axion with an almost massless quark [17], the effect of $D$-particle recoil [18], supersymmetric inflationary models [19], the multidimensional Einstein-Yang-Mills system [20], etc. However interesting, most of these suggestions necessarily involve a quite severe fine tuning of parameters [21]. This fact calls for constructions that allow for a negative deceleration parameter using sources of negative pressure that do not require a potential arising from known particle physics scales. Other difficulties associated with quintessence is that the couplings of the scalar field to matter can lead to observable long-range forces and time variation of fundamental constants of nature [22, 23].

In this work we study the possibility of obtaining the required negative pressure effects by considering a scalar field coupled non-minimally with gravity. It is well known that the nonminimal coupling of fields to gravity have non-trivial implications on issues such as geodesic completeness, stability of the ground state (see [24] and references therein) and the variation of constants of nature. In here, we shall consider the self-interacting Brans-Dicke theory as a prototype of a non-minimally coupled theory and show that accelerated expanding solutions can be obtained and the abovementioned difficulties partially evaded. Actually, accelerated expanding solutions at present time, the so-called scaling attractors, have already been studied in some theories of gravity with non-minimal coupling to a scalar field. Indeed, the nonminimal coupling $\frac{1}{2} \epsilon \phi^{2} R$ has been considered in Ref. 25] with the conclusion that the scalar field behaves as a barotropic fluid, then leading to scaling attractors, only when the constant $\epsilon<<1$ for exponential and power-law potentials. In Ref. [26] the search of scaling attractors 
has been extended to the case of the non-minimal coupling of the form $[1+\epsilon f(\phi)] R$ and $V(\phi)=A f(\phi)^{M}$, where $f(\phi)$ is a power-law or exponential function of the scalar field and $A$ and $M$ are constants, and shown that, in the limit where the kinetic term of the scalar field is dynamically unimportant, the constraint on the time variation of the gravitational coupling is quite severe and limits the fraction of energy in the scalar field to be at most $4 \%$ of the total energy density. We shall see in the next sections that our results, although obtained from somewhat different starting assumptions, are consistent with the conclusions of Refs. [25, 26], namely that although accelerated expanding solutions can be obtained in non-minimally coupled gravity theories the constraint on the variability of the gravitational coupling is quite strong and that it implies a Universe that is considerably older than $H_{0}^{-1}$.

Our starting point is the Brans-Dicke (BD) theory [27] as this is a viable scalar-tensor alternative to General Relativity. In this theory spacetime is described by the interplay of the metric tensor with a scalar field, $\phi$, so that the strength of the gravitational coupling to matter is given by $\phi^{-1}$. The theory is consistent with observational tests which explains the renewed interest in its application both in astrophysics and cosmology. Moreover, the BD theory is also the model arising from string theory at low-energies in the so-called string or Jordan frame and from the dimensional reduction of Kaluza-Klein theories. BD theories and its extensions are known to have relevant cosmological implications [28, 29, 30].

In its simplest version, the salient features of BD theory depend uniquely on the strength of the dimensionless "Dicke coupling constant", $\omega$, that couples the scalar field universally to matter. However, the cosmological setting arising from the simplest BD scenario is, as far as power-law solutions are concerned (cf. equations. below), inconsistent with the cosmic acceleration, unless $|\omega|$ is of order unity. Since the coupling $\omega$ is observationally constrained by solar-system experiments to be $|\omega|>500$ [31], we are led to consider a version of the BD theory where the BD scalar has a potential. For the latter we choose a quadratic self-coupling in the Jordan frame as this implies in a minimal change in the field equations. Thus, our scenario can be regarded as the next to minimal Brans-Dicke model. Furthermore, we show that a negative $\omega$ is required to account for the accelerated expansion of the Universe. 
In what follows we consider an homogeneous and isotropic Friedmann-Robertson-Walker Universe where matter (not including the BD scalar field) is described as a perfect fluid. The relevant thermodynamical fluid variables are the energy density and the isotropic pressure that are related by the equation of state specified above.

\section{Field Equations and Solutions}

The self-interacting BD field theory of our interest is described by the following action:

$$
S=\frac{1}{16 \pi} \int d^{4} x \sqrt{-g}\left[\phi R-\omega \frac{\phi_{, \alpha} \phi_{,}^{\alpha}}{\phi}+V(\phi)\right]+S_{\text {Matter }}
$$

where we choose $V(\phi)=V_{0} \phi^{2}$. As already mentioned in the Introduction a BD theory with a vanishing potential is incompatible with a negative $q_{0}$, at the least for power-law solutions for the scale-factor and scalar field specified below, unless $|\omega|=O(1)$ which is inconsistent with solar system experiments. However, as we shall see in a while, the potential we have introduced for the scalar field changes the equations of motion in a minimal way. It is worth stressing that introducing a potential does not involve, in our proposal, any fine tuning of parameters as our scale, $V_{0}$, will be fixed by the value of Newton's constant and the age of the Universe. Furthermore, as far as giving origin to a time variation of the fundamental constants, our proposal implies indeed in a sizable time variation of the gravitational coupling, even though this is compatible with available data.

From the Lagrangian density (3) we obtain the field equations:

$$
\begin{gathered}
G_{\mu \nu}=\frac{\omega}{\phi^{2}}\left[\phi_{; \mu} \phi_{; \nu}-\frac{1}{2} g_{\mu \nu} \phi_{; \alpha} \phi_{;}^{\alpha}\right]+\frac{1}{\phi}\left[\phi_{; \mu} \phi_{; \nu}-g_{\mu \nu} \square^{2} \phi\right]+\frac{V(\phi)}{2 \phi} g_{\mu \nu}+\frac{8 \pi}{\phi} T_{\mu \nu}^{M a t t e r} \\
\square^{2} \phi=\frac{8 \pi}{3+2 \omega} T^{M a t t e r}+\frac{1}{3+2 \omega}\left[2 V(\phi)-\phi \frac{d V(\phi)}{d \phi}\right] .
\end{gathered}
$$


Hence for our choice of the potential, $V(\phi)$, the last term in Eq. (5) vanishes and $V(\phi)$ affects directly only the dynamics of the scale-factor, $a(t)$.

These field equations in a Friedmann-Robertson-Walker geometry

$$
d s^{2}=-d t^{2}+a(t)^{2}\left[\frac{d r^{2}}{1-k r^{2}}+r^{2} d \Omega^{2}\right],
$$

read

$$
\begin{gathered}
\left(\frac{\dot{a}}{a}\right)^{2}+\frac{k}{a^{2}}=\frac{8 \pi}{3 \phi} \rho-\frac{\dot{a}}{a} \frac{\dot{\phi}}{\phi}+\frac{\omega}{6}\left(\frac{\dot{\phi}}{\phi}\right)^{2}-\frac{V_{0}}{6} \phi \\
3\left(\frac{\ddot{a}}{a}\right)=-\frac{8 \pi}{(3+2 \omega) \phi}[(3+\omega) \rho+3 \omega p]-\omega\left(\frac{\dot{\phi}}{\phi}\right)^{2}+3 \frac{\dot{a}}{a} \frac{\dot{\phi}}{\phi}-\frac{V_{0}}{2} \phi \\
\ddot{\phi}+3 \frac{\dot{a}}{a} \dot{\phi}=\frac{8 \pi}{3+2 \omega}(\rho-3 p)
\end{gathered}
$$

Clearly, these equations must be considered together with the energy conservation equation

$$
\dot{\rho}+3 \frac{\dot{a}}{a}(\rho+3 p)=0
$$

arising from the covariant conservation of the energy-momentum tensor of matter that has been assumed to behave as a perfect fluid.

Finally, the relationship between the gravitational coupling $G(t)$ and the scalar field is given by

$$
G(t)=\left(\frac{2 \omega+4}{2 \omega+3}\right) \frac{1}{\phi(t)}
$$

In order to obtain solutions for the above equations we consider the following power-law form for both scale-factor and scalar field: 


$$
a(t)=a_{0}\left(\frac{t}{t_{0}}\right)^{\alpha} \quad ; \quad \phi(t)=\phi_{0}\left(\frac{t}{t_{0}}\right)^{\beta},
$$

where the zero indices stand for the present time.

Substituting these solutions into Eq. (9) leads, when neglecting the mattter pressure constribution $(\gamma \sim 0)$, to the relationships

$$
3 \alpha=2-\beta \quad ; \quad \beta=\frac{\sigma}{3+2 \omega},
$$

where

$$
\sigma \equiv \frac{8 \pi \rho_{0} t_{0}^{2}}{\phi_{0}}
$$

From Eqs. (11) - (13) and the Hubble parameter at present, $H_{0} \equiv \frac{\dot{a}}{a}\left(t_{0}\right)$, we get the deceleration parameter, $q_{0} \equiv-\frac{\ddot{a} a}{\dot{a}^{2}}\left(t_{0}\right)$, and the time variation of the gravitational coupling:

$$
\begin{aligned}
q_{0} & =\frac{1+\beta}{2-\beta}, \\
\left(\frac{\dot{G}}{G}\right)_{0} & =\frac{3 \beta}{\beta-2} H_{0} .
\end{aligned}
$$

Moreover, adjusting the gravitational coupling to its present value, $G_{N}$, i.e. Newton's constant, we get, after inserting Eq. (11) into Eq. (14) and combining with Eq. (13), for the age of the Universe

$$
t_{0}^{2}=\frac{2 \beta(\omega+2)}{3 \Omega_{M} H_{0}^{2}}
$$

Naturally, for consistency $V_{0}$ must satisfy Eq. (7). Hence, for a flat Universe $(k=0)$ we find

$$
V_{0}=3 G_{N} \Omega_{M} H_{0}^{2}(2 \omega+3)\left[\frac{2 \beta(2 \omega+3)-6 \alpha(\alpha+\beta)+\omega \beta^{2}}{4 \beta(\omega+2)^{2}}\right] .
$$




\section{Conditions for negative $q_{0}$}

In order to have $q_{0}<0$ it is equivalent that the right hand side of Eq. (8) is positive. It is useful then to consider it as a function

$$
f \equiv-\frac{8 \pi}{(3+2 \omega) \phi}[(3+\omega) \rho]-\omega\left(\frac{\dot{\phi}}{\phi}\right)^{2}+3 \frac{\dot{a}}{a} \frac{\dot{\phi}}{\phi}-\frac{V_{o}}{2} \phi
$$

and establish the conditions for having $f>0$.

Power-law solutions Eq. (12) satisfy the field equations only if $\beta=-2$. Then, solving for $\omega$ yields

$$
\omega<-\frac{V_{0} \phi_{0} t_{0}^{2}}{4}-1
$$

Since the product $V_{0} \phi_{0} t_{0}^{2}$ is completely determined by Eqs. (11), (17) and (18) it is easy to see that this condition is satisfied for any value of $\omega$. Therefore, we can conclude that the cosmic accelerated expansion can be driven by a self-interacting Brans-Dicke field as we have specified.

In summary we have, after establishing that $\beta=-2$

$$
\begin{gathered}
a(t)=a_{0}\left(\frac{t}{t_{0}}\right)^{\frac{4}{3}} ; \phi(t)=\phi_{0}\left(\frac{t}{t_{0}}\right)^{-2}, \\
t_{0}^{2}=-\frac{4(\omega+2)}{3 \Omega_{M} H_{0}^{2}}, \\
\left(\frac{\dot{G}}{G}\right)_{0}=\frac{3}{2} H_{0}
\end{gathered}
$$

and

$$
q_{0}=-\frac{1}{4}
$$


We see that the obtained value for $q_{0}$ is consistent with the observations, Eq. (11), and so is the resulting time variation of the gravitational coupling [31], even though many searches are consistent with no variation at all [32]. We stress that, in our proposal, the gravitational coupling is a growing function of time. Morever, notice that, although $f$ is positive independently of the sign of $\omega$, as $\beta$ is negative then in order to have a meaningful age of the Universe it

implies that the Dicke coupling constant must be negative. Interestingly, negative values for $\omega$ are found in the BD effective low-energy models arising from Kaluza-Klein and superstring theories [30]. We shall see in the next section that negative values for $\omega$ are also required for obtaining growing modes for the energy density perturbations in a Universe expanding in an accelerated way.

\section{Perturbed Field Equations and Asymptotic Behaviour}

Having found accelerated expanding solutions in the context of the BD theory we should ask whether the issue of structure formation is modified by the dynamics of the BD scalar field. That is, we are bound to consider the evolution of the energy density perturbations in the context of our BD scenario as these are, of course, associated with the formation of structure in the Universe. Hence in what follows we shall develop a formalism that quickly allows us to find the asymptotic behaviour of the relevant variables for accelerating expanding solutions at present time. This treatment is similar, in its results, to the more encompassing analysis of cosmological perturbations for generalized theories of gravity [33], at least in what concerns the asymptotic behaviour of solutions.

In order to get the matter energy density perturbations we consider the temporal components of Eqs. (8), (9) and (10) after perturbation. Then to obtain the relevant perturbed equations we write the metric tensor as

$$
g_{\mu \nu} \rightarrow g_{\mu \nu}+\delta g_{\mu \nu}
$$


where $\delta g_{\mu \nu}=h_{\mu \nu}$, and work in the gauge $h_{0 \mu}=0$. Our conclusions will be independent of this gauge choice (see [33 for a discussion). For this perturbed metric [34]

$$
\delta R_{00}=\frac{1}{a^{2}}\left[\ddot{h}_{k k}-2 \frac{\dot{a}}{a} \dot{h}_{k k}+2\left[\left(\frac{\dot{a}}{a}\right)^{2}-\frac{\ddot{a}}{a}\right] h_{k k}\right]
$$

The perturbation of the energy-momentum tensor is given by

$$
\delta T^{00}=\delta \rho
$$

and the corresponding trace reads

$$
\delta T=\delta \rho-3 \delta p
$$

For the perturbation of the d'Alembertian of the BD field we have

$$
\delta \square^{2} \phi=\delta \ddot{\phi}+a \dot{a} h^{k k} \dot{\phi}-\frac{1}{2 a^{2}} \dot{h}_{k k} \dot{\phi}+3 \frac{a}{a} \delta \dot{\phi}-\frac{\nabla^{2}}{a^{2}} \delta \phi
$$

The relevant perturbations are parametrized in the following way:

$$
\begin{gathered}
h_{k k}=a^{2} h \quad ; \\
\delta \phi=\lambda \phi \quad, \quad \lambda<<1 ; \\
\delta \rho=\Delta \rho \quad, \quad \Delta<<1,
\end{gathered}
$$

where $h(t), \lambda(t)$ and $\Delta(t)$ are the perturbed gravitational field, scalar field and matter energy density, respectively. 
Since structure is formed when pressure no longer prevents gravitational collapse we set $\gamma=0$. The perturbed equations are then

$$
\begin{gathered}
\frac{1}{2} \ddot{h}+\left(\frac{\dot{a}}{a}\right) \dot{h}=\frac{8 \pi \rho}{\phi} \frac{(2+\omega)}{(3+2 \omega)}(\Delta-\lambda)+\ddot{\lambda}+2(1+\omega) \frac{\dot{\phi}}{\phi} \dot{\lambda}-\frac{V_{o}}{2} \phi \lambda, \\
\ddot{\lambda}+\left(2 \frac{\dot{\phi}}{\phi}+3 \frac{\dot{a}}{a}\right) \dot{\lambda}+\left(\frac{\ddot{\phi}}{\phi}+3 \frac{\dot{a}}{a} \frac{\dot{\phi}}{\phi}\right) \lambda-\frac{1}{2} \frac{\dot{\phi}}{\phi} \dot{\phi}-\frac{\nabla^{2} \lambda}{a^{2}}=\frac{8 \pi \rho}{(3+2 \omega) \phi} \Delta, \\
\dot{\Delta}-\frac{1}{2} \dot{h}+\delta U_{, k}^{k}=0,
\end{gathered}
$$

where $U_{\mu}$ is the comoving fluid velocity.

Inserting solutions (12), (13) with $\beta=-2$ into this set of coupled differential equations we get

$$
\begin{gathered}
\frac{1}{2} \ddot{h}+\frac{\alpha}{t} \dot{h}=\beta(2+\omega) \frac{(\Delta-\lambda)}{t^{2}}+\ddot{\lambda}+2(1+\omega) \frac{\beta}{t} \dot{\lambda}-\frac{V_{0}}{2} \phi_{0}\left(\frac{t}{t_{0}}\right)^{\beta} \lambda \\
\ddot{\lambda}+\left(\frac{\beta+2}{t}\right) \dot{\lambda}+\frac{\beta}{t^{2}} \lambda-\frac{1}{2} \frac{\beta}{t} \dot{h}-\frac{\nabla^{2} \lambda}{a^{2}}=\beta \frac{\Delta}{t^{2}} \\
\dot{\Delta}-\frac{1}{2} \dot{h}+\delta U_{, k}^{k}=0 .
\end{gathered}
$$

In order to continue we suppose that the perturbations behave as plane waves:

$$
\lambda(\vec{r}, t)=\lambda(t) \exp (-i \vec{k} \cdot \vec{r})
$$

where $k$ is the wave number of the perturbation, and we set $\delta U^{k}$ to vanish, which is allowed by an infinitesimal gauge transformation.

Combining then Eq. (36) with (38) we get 


$$
\ddot{\Delta}+\left(\frac{14}{3 t}\right)(\dot{\Delta}-\dot{\lambda})+\left(\frac{6+2 \omega}{t^{2}}\right)(\Delta-\lambda)+\left(\frac{4+4 \omega+\frac{14}{3}}{t}\right) \dot{\lambda}+\frac{V_{0} \phi_{0} t_{0}^{2}}{2 t^{2}} \lambda+\frac{k^{2}}{a_{0}^{2}}\left(\frac{t_{0}}{t}\right)^{\frac{8}{3}} \lambda=0 .
$$

Since we are interested in the asymptotic regime we keep only terms up to $t^{-2}$ and neglect the last term of the previous equation.

Finally, from the value of the product, $V_{0} \phi_{0} t_{0}^{2}=-\left(4 \omega+\frac{20}{3}\right)$ previously obtained, we get the differential equation

$$
\ddot{\Delta}+\frac{C_{1}}{t}(\dot{\Delta}-\dot{\lambda})+\frac{C_{2}}{t^{2}}(\Delta-\lambda)+\frac{C_{3}}{t} \dot{\lambda}+\frac{C_{4}}{t^{2}} \lambda=0
$$

where $C_{1}=\frac{14}{3}$,

$$
\begin{gathered}
C_{2}(\omega) \equiv 6+2 \omega, \\
C_{3}(\omega) \equiv 4(1+\omega)+C_{1},
\end{gathered}
$$

and

$$
C_{4}(\omega) \equiv-\frac{10+6 \omega}{3}
$$

Aiming to solve Eq. (41) we look for solutions of the following form:

$$
\Delta-\lambda=f(t) \quad ; \quad f(t)=\xi t^{\delta} \quad ; \quad \Delta(t)=\chi t^{\theta} \quad
$$

where $\xi$ and $\chi$ are constants.

Inserting these solutions into Eq. (41) we obtain the algebraic equation

$$
\chi \theta^{2}+\left[\chi\left(C_{3}-1\right)+\xi\left(C_{1}-C_{3}\right)\right] \theta+C_{4}(\chi-\xi)+\xi C_{2}=0
$$


and also that $\delta=\theta$.

Solving for $\theta$ we find:

$$
\theta_{ \pm}=\frac{\chi\left(1-C_{3}\right)+\xi\left(C_{3}-C_{1}\right) \pm \sqrt{\left[\chi\left(C_{3}-1\right)+\xi\left(C_{1}-C_{3}\right)\right]^{2}-4 \chi\left[C_{4}(\chi-\xi)+\xi C_{2}\right]}}{2 \chi}
$$

Thus, the existence of growing modes for the energy density perturbations corresponds now to whether there are positive $\theta$ solutions. In the case of our interest, namely $\omega$ negative and $|\omega|>>1$, it is easy to see that

$$
\theta_{ \pm} \rightarrow 2|\omega|\left[\left(1-\frac{\xi}{\chi}\right) \pm \sqrt{\left(1-\frac{\xi}{\chi}\right)^{2}-\frac{1}{2|\omega|}\left(1-2 \frac{\xi}{\chi}\right)}\right]
$$

meaning that $\theta_{+}$corresponds to a growing mode whenever $\xi<\chi$. Moreover, we see that it is only for $\omega$ negative that real $\theta$ solutions always exist. We can then conclude that the cosmological setting of our accelerated expanding solutions does not upset known structure formation scenarios.

\section{Discussion and Conclusions}

In this work we have shown that a minimally extended BD theory with a quadratic self-coupling in the Jordan frame and a negative $\omega$ can account for the accelerated expansion of the Universe yielding $q_{0}=-\frac{1}{4}$. The resulting variation on time of the gravitational coupling is given by $\left(\frac{\dot{G}}{G}\right)_{0}=\frac{3}{2} H_{0}$, being still compatible with data. In this respect our results are similar to the conclusions of Refs. 23, 26] as likewise we find that the existence of scaling attractors in gravity theories with non-minimal coupling to a scalar fields is severly constrained by the time variability of the gravitational coupling. Furthermore, we have also shown that the model allows growing modes for the energy density perturbations of matter implying that the dynamics of the BD field does not upset known structure formation scenarios . 
Before closing, it is worth pointing out some distinct features of our model. The first one being that as accelerated expanding solutions require a negative $\omega$, we then expect the parametrized-post-Newtonian $(\mathrm{PPN})$ parameter $\gamma_{P P N} \equiv \frac{1+\omega}{2+\omega}$ to be, for large $|\omega|$, fairly close but greater than 1. This is a clear observational signature of our proposal, which even though being consistent with current data 35]

$$
\left|\gamma_{P P N}-1\right|<2 \times 10^{-3}
$$

can be, at least in principle, further improved by dedicated solar system experiments.

A second consequence of our proposal is that it implies the Universe is considerably older than $H_{0}^{-1}$. This is incompatible with observation if the age of the Universe is identified with age of the oldest stars, the globular clusters. Contradiction with models of chemical evolution of galaxies may also exist. Even though these constraints are extracted from data assuming a Universe with constant gravitational coupling they point out that the minimal extention of the BD model we have considered is not quite consistent with data. In this respect, it is interesting that the effect of variation on time of the gravitational coupling may reveal itself in the evolution of astrophysical objects. Indeed, as recently discussed [36], stringent bounds on $\omega$

can be set, namely that $\omega>5000$ and that $\left(\frac{\dot{G}}{G}\right)_{0}=O\left(10^{-14}\right)$, based on the luminosity function of white dwarfs assuming that $\dot{G}<0$ and $12.5 \mathrm{Gyr}$ as the age of the Universe. We suspect that this age of the Universe problem is a common feature of all scalar-tensor gravity models that have scaling attractors and do not contain, as for instance in [26], the Einstein-Hilbert term in the action.

Finally, we could conclude remarking that an interesting theoretical challenge would be devicing inflationary models which would, at late times, behave like scalar-tensor gravity theories in what concerns the existence of scaling attractors.

\section{Acknowledgments}

One of us (O.B.) would like to thank Fundação para a Ciência e a Tecnologia (Portugal) for the sabbatical grant BSAB/95/99. 


\section{References}

[1] S. Perlmutter et al., Bull. Am. Phys. Soc. 29 (1997) 1351; astro-ph/9812133; Ap. J. 507 (1998) 46.

[2] A.G.Riess et al., Astron. J. 116 (1998) 1009.

[3] P.M. Garnavich et al., Ap. J. Lett. 493 (1998) 53; Science 279 (1998) 1298; Ap. J. 509 (1998) 74 .

[4] B. Schmidt et al., "The High $-Z$ Supernova Search: Measuring Cosmic Deceleration and Global Curvature of the Universe using type IA Supernovae", astro-ph/9805200.

[5] G. Efstathiou, S.L. Bridle, A.N. Lasenby, M.P. Hobson and R.S. Ellis, "Constraints on $\Omega_{\Lambda}$ and $\Omega_{M}$ from Distant Type 1a Supernovae and Cosmic Microwave Background Anisotropies", astro-ph/9812226.

[6] O. Bertolami, Class. Quantum Gravity 14 (1997) 2748.

[7] V. Sahni and A. Starobinsky, "The Case for a Positive Cosmological $\Lambda$-term", astroph/9904398.

[8] S. Weinberg, Rev. Mod. Phys. 61 (1989) 1;

"Theories of the Cosmological Constant", astro-ph/9610044.

[9] M.C. Bento and O. Bertolami, Gen. Rel. Gravitation 28 (1996) 565;

O. Bertolami and R. Schiappa, Class. Quantum Gravity 16 (1999) 2545.

[10] O. Bertolami, Il Nuovo Cimento 93B (1986) 36; Fortschr. Physik 34 (1986) 829.

[11] R.R. Caldwell, R. Dave and P.J. Steinhardt, Phys. Rev. Lett. 80 (1998) 1582.

[12] M.S. Turner and M. White, Phys. Rev. D56 (1997) R4439.

[13] I. Zlatev, L. Wang and P. Steinhardt, Phys. Rev. Lett. 82 (1999) 896. 
[14] P.J.E. Peebles and B. Ratra, Ap. J. Lett. 325 (1988) 117.

[15] P.G. Ferreira and M. Joyce, Phys. Rev. D58 (1998) 023503.

[16] P. Binétruy, Phys. Rev. D60 (1999) 063502.

[17] J.E. Kim, J. High Energy Phys. 05 (1999) 022.

[18] J. Ellis, N.E. Mavromatos and D.V. Nanopoulos, "Time-Dependent Vacuum Energy Induced by D-Particle Recoil", hep-ph/9810086.

[19] F. Rosati, "Quintessence, Supersymmetry and Inflation", astro-ph/9908518.

[20] M.C. Bento and O. Bertolami, Gen. Rel. Gravitation 31 (1999) 1461.

[21] C. Kolda and D.H. Lyth, Phys. Lett. B458 (1999) 197.

[22] S. Carroll, Phys. Rev. Lett. 81 (1998) 3067.

[23] T. Chiba, Phys. Rev. D60 (1999) 083508.

[24] O. Bertolami, Phys. Lett. B186 (1987) 161.

[25] J.P. Uzan, Phys. Rev. D59 (1999) 123510;

D.J. Holden and D. Wands, "Self-similar cosmological solutions with a non-minimally coupled scalar filed", gr-qc/9908026.

[26] L. Amendola, Phys. Rev. D60 (1999) 043501.

[27] C.H. Brans and R.H. Dicke, Phys. Rev. 124 (1961) 925.

[28] D. La and P.J. Steinhardt, Phys. Rev. Lett. 62 (1989) 376;

D. La, P.J. Steinhardt and E.W. Bertschinger, Phys. Lett. B231 (1989) 231.

[29] M.C. Bento, O. Bertolami and P.M. Sá, Phys. Lett. B262 (1991) 11; Mod. Phys. Lett. A7 (1992) 911 .

[30] S.J. Kolitch and D.M. Eardley, Ann. Phys. (N.Y.) 241 (1995) 128. 
[31] C.M. Will, "Theory and Experiment in Gravitational Physics" (Cambridge University Press, 1993).

[32] G.T. Gillies, Rep. Progr. Phys. 60 (1997) 151.

[33] J. Hwang, Phys. Rev. D53 (1996) 762.

[34] J.P. Baptista, J.C. Fabris and S.V.B. Gonçalves, "Density Perturbations in the BransDicke Theory", gr-qc/9603015.

[35] R.D. Reasenberg et al., Ap. J. Lett. 234 (1979) 219;

R.S. Robertson, W.E. Carter and W.H. Dillinger, Nature 349 (1997) 768.

[36] D.F. Torres, Mod. Phys. Lett. A4 (1999) 1007. 